\title{
Functionally dissociated aspects in anterior and posterior electrocortical processing of facial threat
}

\author{
Dennis J.L.G. Schutter*, Edward H.F. de Haan, Jack van Honk \\ Helmholtz Research Institute, Affective Neuroscience Section, Utrecht University, Heidelberglaan 2, 3584 CS, Utrecht, \\ The Netherlands
}

Received 12 June 2003; received in revised form 1 October 2003; accepted 15 January 2004

Available Online 9 April 2004

\begin{abstract}
The angry facial expression is an important socially threatening stimulus argued to have evolved to regulate social hierarchies. In the present study, event-related potentials (ERP) were used to investigate the involvement and temporal dynamics of the frontal and parietal regions in the processing of angry facial expressions. Angry, happy and neutral faces were shown to eighteen healthy right-handed volunteers in a passive viewing task. Stimulus-locked ERPs were recorded from the frontal and parietal scalp sites. The P200, N300 and early contingent negativity variation (eCNV) components of the electric brain potentials were investigated. Analyses revealed statistical significant reductions in P200 amplitudes for the angry facial expression on both frontal and parietal electrode sites. Furthermore, apart from being strongly associated with the anterior P200, the N300 showed to be more negative for the angry facial expression in the anterior regions also. Finally, the eCNV was more pronounced over the parietal sites for the angry facial expressions. The present study demonstrated specific electrocortical correlates underlying the processing of angry facial expressions in the anterior and posterior brain sectors. The P200 is argued to indicate valence tagging by a fast and early detection mechanism. The lowered N300 with an anterior distribution for the angry facial expressions indicates more elaborate evaluation of stimulus relevance. The fact that the P200 and the N300 are highly correlated suggests that they reflect different stages of the same anterior evaluation mechanism. The more pronounced posterior eCNV suggests sustained attention to socially threatening information.
\end{abstract}

(c) 2004 Elsevier B.V. All rights reserved.

Keywords: Anger; Event-related potentials; Faces; Threat

\section{Introduction}

The selective processing of threatening angry facial expression has been extensively investigated on both the behavioural, functional (e.g. Öhman

*Corresponding author. Tel.: +31-30-2534369; fax: +3130-2534511.

E-mail address: D.Schutter@fss.uu.nl (D.J. Schutter). et al., 2001; Tipples et al., 2002) and physiological level (e.g. Dimberg and Petterson, 2000; Morris et al., 1998). The threatening facial expression of anger provides an important warning signal in the regulation of social hierarchies (Darwin, 1872; Lorenz, 1966; Van Honk and De Haan, 2001). Recently, Van Honk et al. (2000, 2001b) demonstrated attentional biases to angry facial expres- 
sions using a modified emotional Stroop task in healthy volunteers. In this task different colored (i.e. blue, green, yellow or red) angry and neutral faces were presented and the subjects had to name the color of the face as fast as possible. The rationale behind this task was that it would take longer for subjects to name the color of the face when they attended towards an emotional compared to neutral facial expression (i.e. interference). Faster color naming, on the other hand, would suggest that the perceiver was avoiding the processing of the emotional facial expression (i.e. facilitation). Van Honk et al. (2000, 2001b) showed interference during color naming of angry facial expressions, which was interpreted in terms of the face capturing attentional resources.

Although electrophysiological studies have demonstrated specific deflections in the ongoing EEG that are related to facial and affective information processing as early as $100 \mathrm{~ms}$ post-stimulus (e.g. Orozco and Ehlers, 1998; Pizzagalli et al., 1999), these early brain potentials seem to reflect processes related to morphological encoding, such as the extraction of physiognomic features of the face (Bruce and Young, 1986). The N170, for instance, is a negative deflection in the EEG that is specifically involved in the structural face encoding, but does not seem directly sensitive for familiarity or the emotional expression of the face (Balconi and Pozzoli, 2003; Eimer and Holmes, 2002; Herrmann et al., 2002). Pizzagalli et al. (2002), however, recently reported early brain potentials (approx. $160 \mathrm{~ms}$ ), which were a function of affective judgment. Notwithstanding, it has been argued that cognitive and affective information processing arises after $200 \mathrm{~ms}$.

A brain potential which has proven to be sensitive to emotional visual stimulation is a positivity peaking approximately $200 \mathrm{~ms}$, designated as the P200 (Carretié et al., 2001a,b). Horley et al. (2001), for instance, demonstrated reduced P200 amplitude following angry, compared to neutral facial expressions. It has been suggested that the relative early P200 reflects a valence specific orienting index for relevant stimuli. Processes that occur later in the ERP chronometry are argued to be related to more complex cognitive and affectrelated processes. For example, the N300 has been demonstrated to be an emotion-sensitive potential, which is less affected by cognition (Carretié et al., 1997). Carretié et al. (1997) argue that the N300 might reflect an arousal dimension of affective characteristics of visual stimuli. Moreover, the $\mathrm{N} 300$ in the anterior brain region has been positively associated with arousing negative valenced stimuli, whereas a more posterior N300 distribution was related to arousing positive valenced stimuli. In accordance with a frontal-parietal network of attention put forward by Posner and Raichle (1997), the P200 and N300 might be involved in the detection and evaluation of angry facial expressions, respectively. A vigilance network of attention consisting of the frontal and parietal cortex is argued to maintain a state of alertness when salient stimuli are encountered. Angry facial expression are important socially threatening stimuli (Öhman, 1997), hence the involvement of the vigilance network can be assumed, in particular since alertness and action preparation are essential for appropriate responses.

Several studies have reported positive going waveforms after displaying affective pictures (Cuthbert et al., 2000; Keil et al., 2002). Ruchkin et al. (1988) argued that positive going waveforms might particularly be associated with perceptual operations and memory storage, whereas the negative going waveforms are more related to higher order conceptual processing. Cuthbert et al. (2000) suggested that slow waves can be evoked during picture viewing and augmented for emotional laden stimuli, mirroring increases in resource allocation to motivationally relevant incentives. Enhanced late positive going waveforms have indeed been observed after the display of pleasant and unpleasant pictures from the International Affective Picture System (IAPS) (e.g. Cuthbert et al., 2000; Schupp et al., 2000). However, the neural signature of the positive going waveform remains somewhat elusive. In particular negative going waveforms over posterior regions, including the eCNV are assumed to reflect underlying cortical activation through massive depolarisation of apical dendrites (Weisz et al., 2002). Since a slow negative going wave has been found to be indicative of allocation of cortical resources (Altenmüller and Gerloff, 1999) and the CNV in a 
post-stimulus trace reflects selective attention paid to a highly relevant stimulus (Wilkinson and Ashby, 1974), it is feasible that threatening angry facial expressions will elicit an eCNV rather than a positive going waveform. Thus, albeit the eCNV is typically elicited in $\mathrm{S} 1-\mathrm{S} 2$ paradigms, it may nevertheless be conjectured that the eCNV could be evoked and modulated by angry facial expressions, indexing a rudimentary form of action preparation.

Although, the electrophysiology of face processing has been abundantly investigated, little is known about the processing of angry faces in the fronto-parietal network of attention. The present study was designed to investigate the temporal dynamics of the frontal and parietal EEG displaying angry compared to neutral and happy facial expressions. As noted, the P200 amplitude is argued to indicate valence tagging and amplitude reductions have been observed after displaying angry facial expressions (Horley et al., 2001). Thus, currently reductions in P200 amplitude were hypothesized. Larger N300 amplitude indicating elaborate attentional processing were hypothesized to occur after the angry facial expression. Also, since the eCNV varies as a function of alertness and sustained attention, a more pronounced negativity for the threatening angry facial expression was expected. If indeed the P200, N300 and eCNV amplitude would be modulated by the threatening angry facial expression, it would be worthwhile to investigate possible functional relationships between the components as well.

\section{Methods}

\subsection{Participants}

Eighteen healthy volunteers (mean \pm S.D. age, $21.6 \pm 1.9$ years) were recruited among students at Utrecht University, Utrecht, The Netherlands. Participants (11 females) were non-smoking and right-handed as indexed by the Edinburgh Handedness Inventory (Oldfield, 1971) (mean \pm S.D. score, $44.3 \pm 3.2$ ). None of the subjects had a history of psychiatric or neurological conditions and had normal or corrected-to-normal vision. All volunteers were unaware of the aim of the study.
Informed consent was obtained and participants received payment for taking part in the study. The protocol was approved by the local ethical committee of Faculty of Social Sciences.

\subsection{Passive viewing task}

The stimuli were taken from Ekman and Friesen (1976) Pictures of Facial Affect and other comparable specially prepared facial stimuli (Van Honk et al., 2000). Pictures of 10 different individuals each displaying angry, neutral and happy facial expression were used. Happy facial expressions were included to control for valence (Van Honk et al., 2001a). The passive viewing task consisted of ninety consecutive non-paced $750 \mathrm{~ms}$ presentations of thirty facial expressions of each category. Prior to each sequence of facial picture, a fixation cross appeared at the centre of the screen for $750 \mathrm{~ms}$. The inter-stimulus interval between the presentations varied randomly between the 1500 and $2500 \mathrm{~ms}$. All stimuli were projected in the centre of the screen in grey-scales on a black background, using a $70 \mathrm{~Hz}$ computer screen at a distance of $110 \mathrm{~cm}$ from the eyes. The image sizes were $14 \times 9 \mathrm{~cm}$ and the vertical and horizontal visual angles being $3.64^{\circ}$ and $2.34^{\circ}$, respectively. The stimuli were presented in a random fashion.

\subsection{Event related potentials}

EEGs were recorded from eight electrodes, i.e. F3, F4, P3, P4, Fz, Cz, Pz and Oz scalp positions, using an Electro-Cap with $\mathrm{Ag} / \mathrm{AgCl}$ electrodes (Neurosoft, Inc.) and according to the International 10-20 System. EEG signals were referenced to an electrode placed behind the subject's right mastoid. The choice of the reference has been a longstanding issue in electrophysiological studies (see for instance Nunez et al., 1997; Picton et al., 2000; Reilly, 1999). Various solutions have been proposed in order to minimize the influence of reference, but no agreement on an optimal solution for the reference problem has been reached yet (Hagemann et al., 2001). The mastoid reference is often used to maximize inter-electrode distance and to avoid mixed activity from two different scalp areas. This montage is based on the assumption 
that unlike, for instance, vertex referencing the mastoid site picks up no or minimal electrical activity. Although, the reference electrode ought to be 'silent', this is hard to accomplish. One of the solutions is to use a scalp site with minimal activity (Hagemann et al., 1998). Furthermore, different reference strategies do not necessarily compromise whether and where the ERPs are recorded. For instance, Horley et al. (2001) ran separate analyses with two different reference montages with no unduly effects on the P200 component. Finally, Keil et al. (2002) recently used a high-density electrode (129-sensor) array and an average reference to investigate affective picture processing. Surprisingly, the polarity and topography of the effects were similar to that observed in previous comparable work, which had used smaller montage sizes and linked mastoids.

For the purpose of artefact scoring, vertical (VEOG) and horizontal (HEOG) eye movements were recorded. $\mathrm{Ag} / \mathrm{AgCL}$ electrode pairs (bipolar) were placed at the supra- and suborbit of the right eye and at the external canthi of each eye. ECI EEG Gel was used for both EEG and EOG and all electrode impedances were less than $5000 \Omega$. An acquisition amplifier (Ampligraph) was used to filter incoming signals (low-pass cut-off frequency $70 \mathrm{~Hz}$; time constant $3 \mathrm{~s}$ ). For the EEG recordings NeuroScan software (El Paso, Texas) was used. Amplification was set at 20000 for both the EEG and EOG leads, and the sample rate was $250 \mathrm{~Hz}$. Raw EEG was digitally low-pass filtered offline with a cut-off frequency of $15 \mathrm{~Hz}$, corrected for eye-movements, using linear regression, and epoched in a -50 to $1024 \mathrm{~ms}$ stimulus-locked time window. Muscular and other sources of artefacts $( \pm 50 \mu \mathrm{V})$ in the stimulus-locked epochs were removed prior to further analysis. The designation of an artefact in one of the leads resulted in removal of that epoch for all channels in order to ensure that the remaining data were identical for all sites in time. The remaining EEG epochs were baseline corrected using the mean amplitude $50 \mathrm{~ms}$ prior to stimulus onset and averaged across the different facial expressions, constructing a mean ERP for the angry, neutral and happy facial expressions for the passive viewing task. An averaged waveform from at least 25 artefact-free trials for each emotional facial expression could be obtained.

\subsection{Procedure}

Upon arrival at the psychological laboratory volunteers written informed consent was obtained and the computerized version of the Edinburgh Handedness Inventory was completed. Next, participants were prepared for the EEG recording. After completion, the subjects were seated in a comfortable chair situated in a dimly lit room adjacent to the control chamber. EEG was recorded during passive viewing task, and subjects were instructed to relax, keep head movements to a minimum, and to focus on the screen. The entire experiment took approximately $1 \mathrm{~h}$ per subject.

\subsection{Data analyses}

For each individual the average amplitude of the P200 and N300 components for each facial expression category were determined by means of peak-amplitude scoring between 150-250 ms and 250-350 ms time intervals, respectively, (adapted from Carretié and Iglesias, 1995). The slow wave activity, the eCNV, was defined as the mean amplitude in the $425-525 \mathrm{~ms}$ time window. To investigate anterior-posterior and interhemispheric differences, separate $3 \times 3$ MANOVAs for the frontal $(\mathrm{F} 3, \mathrm{~F} 4, \mathrm{Fz})$ and parietal $(\mathrm{P} 3, \mathrm{P} 4, \mathrm{Pz})$ were performed with Electrode and Valence (angry, neutral and happy facial expressions) as withinsubject factors and the mean amplitude of the P200, N300 components and the eCNV as the dependent variables. Potential functional relationship between the brain potentials of interest and emotional face processing were investigated using post-hoc Pearson's correlations. The alpha level of significance (two-tailed) was set at 0.05 throughout.

\section{Results}

Separate MANOVAs for the frontal and parietal electrode sites revealed a significant main Valence effect of reduced P200 amplitude for the angry facial expressions compared to both the anterior 
$[F(2,16)=3.6: \quad P<0.05]$ and posterior regions $[F(2,16)=5.0: P=0.02]$. Two separate MANOVAs were conducted to test for differences between frontal and parietal distribution of the N300. There was an augmentation of the N300 amplitude over the frontal electrodes $[F(2,16)=4.4: P=0.03]$, but the effect failed to reach significance over the posterior sites $[F(2,16]=2.2: P>0.15]$ for the angry facial expression. Two separate MANOVAs yielded a significance larger eCNV over the parietal $[F(2,16)=5.1: P<0.02]$, but not the frontal electrode sites for the angry facial expression $[F(2,16)=1.9: P>0.17]$. The parietal eCNV and its reactivity seem to be in accordance with the typical topographical distribution of the eCNV over the scalp. Fig. 1 displays the grand-averaged ERPs for the angry, neutral and happy facial expressions over the frontal and parietal electrode sites. Finally, Pearson correlations demonstrated a highly significant relationship between the anterior P200 and N300 amplitude for the angry facial expression $[r(18)=0.8: \quad P=0.0003]$, which is shown in Fig. 2. An association between P200 and eCNV, was however not found $[r(18)=0.1: P=$ $0.65]$.

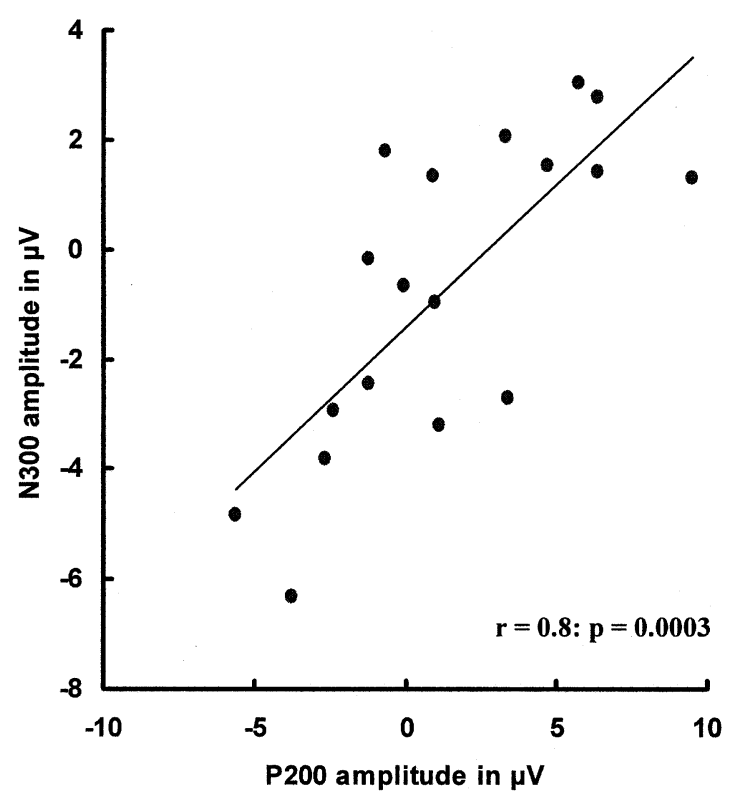

Fig. 2. The anterior P200-N300 amplitude correlation for the angry facial expressions.
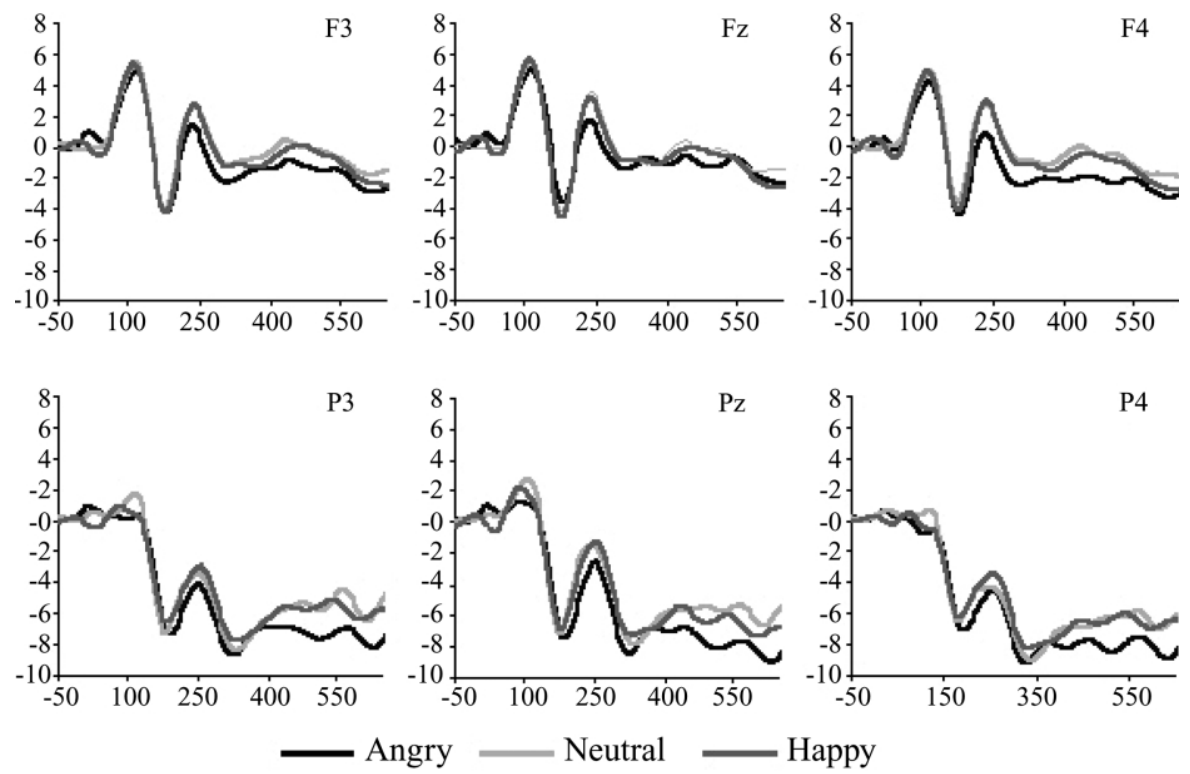

Fig. 1. Mean scalp-recorded ERPs in microvolt for the angry, happy and neutral facial expressions. 


\section{Discussion}

The present study investigated the electrocortical processes over the anterior and posterior brain regions for angry, neutral and happy facial expressions.

Unlike most of the earlier brain potentials the P200 has demonstrated to be valence specific (Carretié et al., 2001a). In accordance with this notion, a recent study by Horley et al. (2001) indeed showed a relationship between reduced P200 amplitude following the presentation of angry, compared to neutral facial expressions, which was replicated in the present study. Sokolov (1975) suggested that in the case of threatening stimuli the cortical analysers are shut down, in order to preclude higher level cognitive processing to interfere with the need for efficient and quick responses. The available energetic resources must immediately be re-allocated to (subcortical) brain structures involved in the preparation of fightflight responses (Ledoux, 2002). In the present respect, it might thus be argued that the reduced P200 component reflects a relative fast and early threat related index of information processing: a global valence tagging or detection system. A similar although reversed pattern was observed for the N300 which showed to be larger for the angry facial expressions. Carretié et al. (1997, 2001a) already emphasized the importance of this negative brain potential in the processing of emotionally salient stimuli. Alternatively, Rockstroh et al. (1989) and Schupp et al. (1994) have argued that positive and negative brain potentials relate to inhibitory and facilitatory processes. Accordingly, the reduced P200 amplitude would stand for cortical activation and enhanced processing which might explain the physiological relation with the enhanced N300 amplitude.

Notably, in the present study the N300 amplitude appeared to be significantly modulated over the anterior brain sectors. This observation is in accordance with the findings of Carretie et al. (1997) who showed that the N300 which most pronounced over the frontal cortex for arousing negative related stimuli. In the present respect, it is argued that the N300 reflects a more in depth evaluation of the relevant stimuli, which have passed the initial detection of significance (the P200). It is furthermore interesting to observe that besides from being modulated by the angry facial expression, the P200 was also positively associated with the emergence of the N300. This provides evidence for a relationship between the computations underlying detection and evaluation. This P200-N300 complex is likely part of an anterior network involved in the detection and evaluation of threat. The well-documented executive functions of the frontal brain areas in cognition and emotion (Fuster, 1997) are in support of this assumption.

Parietal enhancements of the eCNV for angry, but not neutral and happy faces were observed in the current study. Cuthbert et al. (2000) and Keil et al. (2002) and Schupp et al. (2003) have reported positive going waveforms following emotional pictures. However, in the present study a different set of emotional stimuli was used. The average ERP of their negative IAPS pictures is not exclusively based on emotional facial expressions, since the IAPS also uses rather gruesome negative pictures of, for instance, mutilated bodies (e.g. Cuthbert et al., 2000). This dissimilarity might well evoke a different cascade of electrophysiological effects. The eCNV is assumed to reflect underlying cortical activity through massive depolarisation of apical dendrites (Weisz et al., 2002) and suggested to function as a warning-type signal (Weerts and Lang, 1973). Interestingly as can be read from the Introduction, Wilkinson and Ashby (1974) suggested that the CNV in a poststimulus time period might index selective attention. The parietal lobules have been implicated in a vigilant attention network (Posner and Raichle, 1997). Adding evidence to this parietal involvement in emotional processing, Schutter et al. (2002) recently showed that baseline parietal activity was predictive for selective attention to the angry facial expressions. Since no correlation was found between the parietal P200 and the eCNV, it can be argued that the parietal eCNV reflects a separate functional process, instantiated after the completion of the first evaluation stage. Thus, the eCNV might be associated with sustained attention and action preparation. It can nevertheless be argued that the selective processing of the angry facial expression is primarily a consequence of 
arousal (Cuthbert et al., 2000; Keil et al., 2002; Schupp et al., 2000). At least subjectively, the emotional expressions of anger and happiness do not seem to differ on the arousal dimension. Indeed, Balconi and Pozzoli (2003) recently found that the angry and the happy facial expressions are rated as negative and positive in valence, but both highly arousing. We did not ask the participants to subjectively rate the arousal dimension of the faces nor did we apply autonomic measures. Presently, the angry face did exert selective modulatory effects on the N300 and the P200 component. Note that the N300 has been linked to negatively arousing events (Carretié et al., 1997), whereas the reduced P200 amplitude has specifically been associated with angry facial expressions (Horley et al., 2001). On the basis of above-noted findings (Balconi and Pozzoli, 2003; Horley et al., 2001) and the current differential findings on angry and happy faces, it can be suggested that valence is involved in the ERP of the angry facial expression.

A possible limitation of the study is the small number of electrode sites from which EEG was recorded. Larger montage-sizes might provide more detailed information regarding polarity and topographical distribution, albeit the parsimonious use of less dense arrays does not compromise findings with respect to polarity and topographical distribution per se. In defense, Keil et al. (2002) recently used a high-density electrode (129-sensor) array and average referencing and demonstrated that the polarity and topography of ERP components were similar to that observed in previous work with smaller montage sizes and linked mastoids. It should, however, be noted that larger electrode arrays do provide better estimates of topographical distribution enabling electrocortical source analysis, this was beyond the scope of our study.

In sum, based on the present data, it is concluded that the frontal brain sector is involved in the detection (P200) and evaluation (N300) of angry facial expressions, whereas the posterior cortices are involved in sustained selective attention and action preparation (eCNV). The frontal P200N300 complex and parietal eCNV may be part of a functionally dissociated fronto-parietal network of attention in which anterior and posterior sectors are responsible for different aspects of affective processing.

\section{Acknowledgments}

This study was supported by an Innovational Research Grant (\# 016-005-060) from the Netherlands Organization for Scientific Research (NWO).

\section{References}

Altenmüller, E.O., Gerloff, C., 1999. Psychophysiology and the EEG. In: Niedermeyer, E., Lopes da Silva, F. (Eds.), Electroencephalography: Basic Principles, Clinical Applications, and Related Fields. 4th ed. Williams and Wilkins, Baltimore, pp. 637-655.

Balconi, M., Pozzoli, U., 2003. Face-selective processing and the effect of pleasant and unpleasant emotional expressions on ERP correlates. Int. J. Psychophysiol. 49, 67-74.

Bruce, V., Young, A., 1986. Understanding face recognition. Br. J. Psychol. 77, 305-327.

Carretié, L., Iglesias, J., 1995. An ERP study on the specificity of facial expression processing. Int. J. Psychophysiol. 19, 183-192.

Carretié, L., Iglesias, J., Garcia, T., 1997. A study of emotional processing of visual stimuli through event-related potentials. Brain Cogn. 34, 207-217.

Carretié, L., Mercado, F., Tapia, M., Hinojosa, J.A., 2001a. Emotion, attention, and the 'negativity bias', studies through event-related potentials. Int. J. Psychophysiol. 41, 75-85.

Carretié, L., Martin-Loeches, M., Hinojosa, J.A., Mercado, F., 2001b. Emotion and attention interaction studied through event-related potentials. J. Cogn. Neurosci. 13, 1109-1128.

Cuthbert, B.N., Schupp, H.T., Bradley, M.M., Birbaumer, N., Lang, P.J., 2000. Brain potentials in affective picture processing: covariation with autonomic arousal and affective report. Biol. Psychol. 52, 95-111.

Darwin, C., 1872. The Expression of the Emotions in Man and Animals. J. Murray, London.

Dimberg, U., Petterson, M., 2000. Facial reactions to happy and angry facial expressions: evidence for right hemisphere dominance. Psychophysiology 37, 693-696.

Eimer, M., Holmes, A., 2002. An ERP study on the time course of emotional face processing. Neuroreport 13, 427-431.

Ekman, P., Friesen, W., 1976. Pictures of Facial Effect. Consulting Psychologist Press, Palo Alto, CA.

Fuster, J.M., 1997. The Prefrontal Cortex: Anatomy, Physiology, and Neuropsychology of the Frontal Lobe. LippincottRaven Publishers, New York.

Hagemann, D., Naumann, E., Becker, G., Maier, S., Bartussek, D., 1998. Frontal brain asymmetry and affective style: a conceptual replication. Psychophysiology 35, 372-388. 
Hagemann, D., Naumann, E., Thayer, J.F., 2001. The quest for the EEG reference revisited: a glance from brain asymmetry research. Int. J. Psychophysiol. 38, 847-857.

Herrmann, M.J., Aranda, D., Ellgring, H., Mueller, T.J., Strik, W.K., Heidrich, A., et al., 2002. Face-specific event-related potential in humans is dependent from facial expression. Int. J. Psychophysiol. 45, 241-244.

Horley, K., Gonsalvez, C., Williams, L., Lazzaro, I., Bahramali, H., Gordon, E., 2001. Event-related potentials to threatrelated faces in schizophrenia. Int. J. Neurosci. 107, 113-130.

Keil, A., Bradley, M.M., Hauk, O., Rockstroh, B., Elbert, T., Lang, P.J., 2002. Large scale neural correlates of affective picture processing. Psychophysiology 39, 641-649.

Ledoux, J.E., 2002. The Synaptic Self. Simon and Schuster, New York.

Lorenz, K., 1966. On Aggression. Harcourt, Brace and World, New York.

Morris, J.S., Öhman, A., Dolan, R.J., 1998. Conscious and unconscious emotional learning in the human amygdala. Nature 393, 467-470.

Öhman, A., 1997. A fast blink of the eye: evolutionary prepardness for preattentive processing of threat. In: Lang, P.J., Simons, R.F., Balaban, M.T. (Eds.), Attention and orienting: Sensory and Motivational Processing. Laurence Earlbaum Associates Hillsdale, New Jersey, pp. 165-184.

Öhman, A., Lundqvist, D., Esteves, F., 2001. The face in the crowd revisited: a threat advantage with schematic stimuli. J. Pers. Soc. Psychol. 80, 381-396.

Oldfield, R.C., 1971. The assessment and analysis of handedness: the Edinburgh inventory. Neuropsychologia 9, 97-113.

Orozco, S., Ehlers, C.L., 1998. Gender differences in electrophysiological responses to facial stimuli. Biol. Psychiatry 44, 281-289.

Nunez, P.L., Srinivasan, R., Westdorp, A.F., Wijesinghe, R.S., Tucker, D.M., Silberstein, R.B., et al., 1997. EEG coherency. I: statistics, reference electrode, volume conduction, Laplacians, cortical imaging, and interpretation at multiple scales. Electroencephalogr. Clin. Neurophysiol. 103, 499-515.

Picton, T.W., Bentin, S., Berg, P., Donchin, E., Hillyard, S.A., Johnson Jr, R., et al., 2000. Guidelines for using human event-related potentials to study cognition: recording standards and publication criteria. Psychophysiology 37, 127-152.

Pizzagalli, D., Regard, M., Lehmann, D., 1999. Rapid emotional face processing in the human right and left brain hemispheres: an ERP study. Neuroreport 10, 2691-2698.

Pizzagalli, D.A., Lehmann, D., Hendrick, A.M., Regard, M., Pascual-Marqui, R.D., Davidson, R.J., 2002. Affective judgments of faces modulate early activity $(\sim 160 \mathrm{~ms})$ within the fusiform gyri. Neuroimage 16, 663-677.

Posner, M.I., Raichle, M.E., 1997. Images of Mind. Scientific American Library, New York.

Reilly, E.L., 1999. EEG recording and operation of the apparatus. In: Niedermeyer, E., Lopes da Silva, F. (Eds.),
Electroencephalography: Basic Principles, Clinical Applications, and Related Fields. 4th edn. Williams and Wilkins, Baltimore, pp. 122-142.

Rockstroh, B., Elbert, T., Canavan, A., Lutzenberger, W., Birbaumer, N., 1989. Cortical Potentials and Behaviour. 2nd edn. Urban and Schwarzenberg, Baltimore.

Ruchkin, D.S., Johnson, R., Mahaffey, D., Sutton, S., 1988. Toward a functional categorization of slow waves. Psychophysiology 25, 339-353.

Schupp, H.T., Lutzenberger, W., Rau, H., Birbaumer, N., 1994. Positive shifts of event-related potentials: a state of cortical disfacilitation as reflected by the startle reflex probe. Electroencephalogr. Clin. Neurophysiol. 90, 135-144.

Schupp, H.T., Cuthbert, B.N., Bradley, M.M., Cacioppo, J.T., Ito, T., Lang, P.J., 2000. Affective picture processing: the late positive potential is modulated by motivational relevance. Psychophysiology 37, 257-261.

Schutter, D.J.L.G., Putman, P., Hermans, E.J., Van Honk, J., 2002. Parietal electroencephalogram beta asymmetry and selective attention to angry facial expressions in healthy human subjects. Neurosci. Lett. 314, 13-16.

Sokolov, E.N., 1975. The neuronal mechanisms of the orienting reflex. In: Sokolov, E.N., Vinogradova, O.S. (Eds.), Neuronal Mechanisms of the Orienting Reflex. Laurence Earlbaum Associates Hillsdale, New Jersey, pp. 217-338.

Tipples, J., Atkinson, A.P., Young, A.W., 2002. The eyebrow frown: a salient social signal. Emotion 2, 288-296.

Van Honk, J., Tuiten, A., Van den Hout, M., Koppeschaar, H., Thijssen, J., De Haan, E., et al., 2000. Conscious and preconscious selective attention to social threat: different neuroendocrine response patterns. Psychoneuroendocrinology $25,577-591$.

Van Honk, J., Tuiten, A., Hermans, E., Putman, P., Koppeschaar, H., Thijssen, J., et al., 2001a. A single administration of testosterone induces cardiac accelerative responses to angry faces in healthy young women. Behav. Neurosci. 115, 238-242.

Van Honk, J., Tuiten, A., Van den Hout, M., De Haan, E.H.F., Stam, H., 2001b. Attentional biases for angry faces: relationships to trait anger and anxiety. Cognition Emotion 15, 279-297.

Van Honk, J., De Haan, E.H.F., 2001. Conscious and unconscious processing of emotional faces. In: De Gelder, B., De Haan, E.H.F., Heywood, C.A. (Eds.), Out of Mind: Varieties of Unconscious Processes. Oxford University Press, New York, pp. 222-237.

Weerts, W.G., Lang, P.J., 1973. The effects of eye fixation and stimulus and response location on the contingent negativity variation (CNV). Biol. Psychol. 1, 1-19.

Weisz, N., Schandry, R., Jacobs, A., Mialet, J., Duschek, S., 2002. Early contingent negative variation of the EEG and attentional flexibility are reduced in hypotension. Int. J. Psychophysiol. 45, 253-260.

Wilkinson, R.T., Ashby, S.M., 1974. Selective attention, contingent negative variation and the evoked potential. Biol. Psychol. 1, 167-179. 